\title{
MODELO HETEROGÊNEO DE CARBONO ATIVADO PARA CARACTERIZAÇÃO TEXTURAL
}

\author{
P. F. G. SILVINO ${ }^{1}$, R. V. GONÇALVES ${ }^{1}$, M. A. G. PAIVA ${ }^{1}$, S. M. P. LUCENA ${ }^{1}$ \\ ${ }^{1}$ Universidade Federal do Ceará, Departamento de Engenharia Química. \\ E-mail para contato: pedro@gpsa.ufc.br
}

\begin{abstract}
RESUMO - A aplicabilidade de amostras de carbono ativado está intimamente ligada às suas características superficiais. Técnicas para caracterizar materiais nanoporosos através de simulação molecular apresentam grande aplicação, contudo, a estrutura molecular do carbono ativado não é bem definida. Abordagens clássicas aproximam um poro de carbono ativado por uma fenda de placas paralelas de grafeno. Os chamados modelos virtuais de carbono condensado adicionam fatores de heterogeneidade aos modelos clássicos, representando melhor a estrutura molecular do carbono ativado. Foram caracterizadas seis amostras de carbono ativado com diferentes graus de ativação e de diferentes materiais precursores. Utilizou-se inicialmente um modelo clássico de geometria slit-pores, em seguida foi proposto um modelo heterogêneo contendo imperfeições superficiais. Observou-se que os ajustes obtidos com o modelo clássico apresentou uma média de erros residuais de 6,94, considerando as seis amostras, enquanto que o modelo heterogêneo gerou uma média de erros de apenas 0,54. Com isso fica clara a validade do modelo proposto.
\end{abstract}

\section{INTRODUÇÃO}

As características texturais de uma amostra de carbono ativado definem sua aplicação. Estas características dependem da natureza do material precursor e do processo de ativação ao qual foi submetido. A distribuição de tamanhos de poros (PSD, do inglês: Pore Size Distribution) é a principal informação textural de um CA. Técnicas para extrair a PSD a partir da isoterma de adsorção via simulação molecular vem sendo utilizadas com êxito em substituição aos modelos fenomenológicos (Davies et al., 1999). Para realizar a simulação molecular de CA, deve-se definir o seu modelo molecular adequado. A estrutura do CA não é bem definida, podendo ser compreendida como uma combinação de poros formados por folhas de grafeno, interconectados e distribuídos aleatoriamente (Marsh e Rodríguez-Reinoso, 2006).

Uma forte tendência atual é a de aproximar os modelos moleculares às condições reais da amostra. Os chamados modelos virtuais de carbono condensados - VPC - (Biggs e Buts, 2006) vão além do modelo convencional de fendas paralelas de folhas de grafeno e buscam incorporar efeitos de heterogeneidade geométrica, topológica e química que caracterizam os carbonos ativados reais (Davies et al., 1999; Lucena et al., 2010). O presente estudo tem como objetivo o aperfeiçoamento do procedimento de caracterização textural via simulação molecular de carbonos ativados através do desenvolvimento de um modelo virtual de carbono condensado que levam em conta fatores de heterogeneidade superficiais. 


\section{METODOLOGIA}

\subsection{Métodos Computacionais}

Caracterização Textural: O procedimento para caraterização textural de amostras de CA, via simulação molecular tem início na confecção de uma coleção de isotermas de adsorção simuladas, chamada de kernel de isotermas locais, ou simplesmente kernel. Cada isoterma local está associada a um tamanho de poro específico, representando uma amostra hipotética de CA que apresenta um único tamanho de poro. Uma amostra de CA real pode ser aproximada por uma combinação de amostras hipotéticas de tamanhos de poros homogêneos. Como consequência, sua isoterma experimental de um gás sonda pode ser aproximada por uma isoterma total, que é a soma das isotermas locais do referido gás, ponderada pelo volume de poros, conforme a Equação 1. O presente estudo utilizou nitrogênio a $77 \mathrm{~K}$ como gás sonda.

$$
Q_{T}(p)=\int q(p, H) \cdot f(H) \cdot d H
$$

Onde " $Q_{T}(p)$ " representa a isoterma total, " $p$ " assume os valores das pressões da isoterma de adsorção, " $q(p, H)$ " representa as isotermas locais e " $H$ " assume valores de tamanhos dos poros. O único dado desconhecido até então é o termo " $f(H)$ ", que contém a função de distribuição de tamanhos de poros (PSD). O objetivo é achar os valores de " $f(H)$ " que tornem a isoterma total " $Q_{T}(p)$ " o mais próximo possível da isoterma simulada " $Q_{E X P}(p)$ ". A deconvolução deste sistema é feita através do método de mínimos quadrados não negativos, com fator de suavização proposto por Davies et al. (1999). A função objetivo é dada pela Equação 2 e o valor do parâmetro de regularização " $\alpha$ " é determinado previamente de acordo com a metodologia proposta por Wilson (1992).

$$
\text { erro }=\sum\left(Q_{E X P}(p)-Q_{T}(p)\right)^{2}+\alpha \cdot \sum\left(\frac{\partial^{2} f(H)}{\partial H^{2}}\right) \rightarrow \min
$$

Simulação Molecular: As isotermas locais de adsorção são obtidas utilizando o método de Monte Carlo no ensemble Grande Canônico - GCMC (Allen e Tildesley, 1991). Foi utilizado o modelo átomo-unitário para o nitrogênio (Lucena et al., 2010). As interações intermoleculares foram modeladas pelo potencial 12-6 de Lennard-Jones, Equação 3. Os parâmetros de campo de força utilizados encontram-se organizados na Tabela 1. As simulações foram conduzidas no software comercial de simulação molecular: Materials Studio, da fabricante Accelrys.

$$
U\left(r_{i j}\right)=4 \varepsilon_{i j}\left[\left(\frac{\sigma_{i j}}{r_{i j}}\right)^{12}-\left(\frac{\sigma_{i j}}{r_{i j}}\right)^{9}\right]
$$

Tabela 1 - Parâmetros do potencial de LJ para o $\mathrm{N}_{2}$ em CA

\begin{tabular}{cccc}
\hline \multicolumn{2}{c}{ Parâmetros fluido-fluido (ff; $\left.\mathrm{N}_{2}-\mathrm{N}_{2}\right)}$. & \multicolumn{2}{c}{ Parâmetros sólido-fluido (sf; C-N $\left.)_{2}\right)}$. \\
\hline$\sigma_{\mathrm{ff}}(\AA)$ & $\varepsilon_{\mathrm{ff}} / \mathrm{k}(\mathrm{K})$ & $\sigma_{\mathrm{sf}}(\AA)$ & $\varepsilon_{\mathrm{s}} / \mathrm{k}(\mathrm{K})$ \\
\hline 3,62 & 101,5 & 3,494 & 56,4 \\
\hline
\end{tabular}

Lucena et al. (2010); Constante de Boltzman, $\mathrm{k}=1,3806503 \cdot 10^{-23} \mathrm{~J} / \mathrm{K}$ 


\subsection{Modelo Virtual de Carbono Ativado}

O modelo virtual clássico de um poro de carbono ativado baseia-se na geometria slit (Tan e Gubbins, 1992), em que um poro é aproximado por uma fenda formada pelo empilhamento de folhas paralelas e perfeitas de grafeno. O modelo heterogêneo proposto baseia-se no modelo desenvolvido por Seaton et al. (1997). Concebido a partir da consideração de existam imperfeições superficiais no poro real. Estas imperfeições foram incorporadas ao modelo através da remoção aleatória de átomos de carbono das camadas superficiais do poro.

A Figura 1 traz os quatro níveis de imperfeições utilizados no modelo heterogêneo: 0, 25, 50 e $75 \%$ de remoção dos átomos que compõem a superfície do poro. Este modelo será referido no presente trabalho como modelo de poros atacados, em menção ao termo utilizado por Seaton et al. (1997): randomly etched graphite. Um poro cuja metade dos átomos de carbono da superfície foi removida, por exemplo, será referenciado como $50 \%$ atacado.

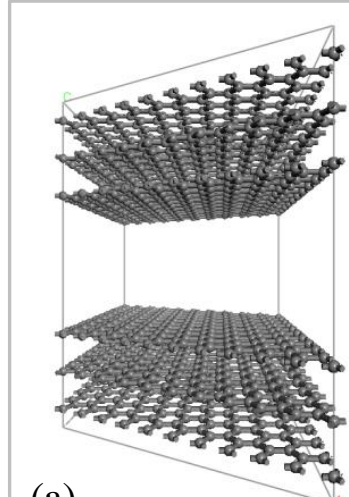

(a)

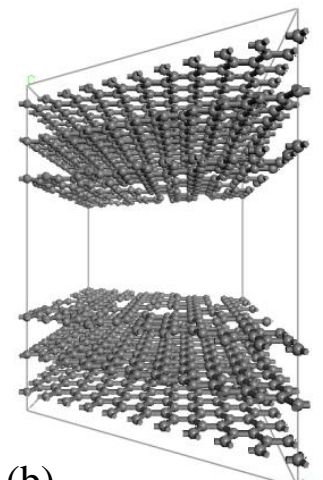

(b)

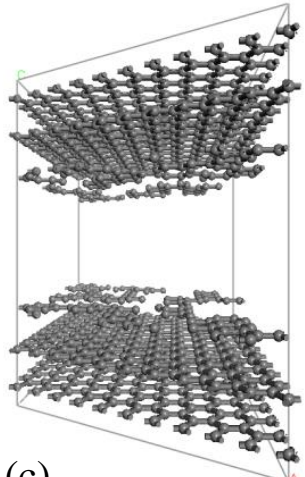

(c)

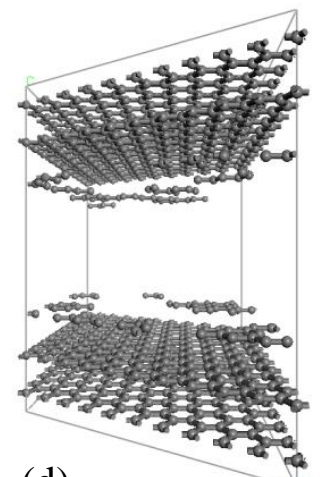

(d)

Figura 1 - Modelos de poros de CA: poro perfeito (a), $25 \%$ (b), $50 \%$ (c) e $75 \%$ (d) atacados.

Foram utilizados vinte e dois tamanhos de poros para gerar as coleções de isotermas locais, conforme a Tabela 2, cada tamanho de poro com os quatro níveis de ataque. Uma primeira coleção (kernel-A) será montada apenas com as vinte e duas isotermas geradas para os poros perfeitos ( $0 \%$ de ataque). Uma segunda coleção será montada com as isotermas de todos os oitenta e oito modelos virtuais de poros (kernel-B).

Tabela 2 - Tamanhos de poros, Hcc, presentes nos kernels

\begin{tabular}{ccccccccccc}
\hline \multicolumn{10}{c}{ Tamanhos de poros - Hcc $(\AA)$} \\
\hline 7,00 & 8,20 & 9,70 & 11,50 & 13,90 & 16,60 & 20,50 & 25,10 & 31,00 & 38,30 & 47,50 \\
7,60 & 8,90 & 10,70 & 12,50 & 15,20 & 18,50 & 22,70 & 27,90 & 34,40 & 42,70 & 53,00 \\
\hline
\end{tabular}

\section{RESULTADOS E DISCUSSÕES}

\subsection{Kernel de Isotermas Locais}

A Figura 2 traz algumas das isotermas locais geradas para compor os kernels A e B. Estas isotermas foram analisadas e apresentaram-se todas linearmente independentes. 

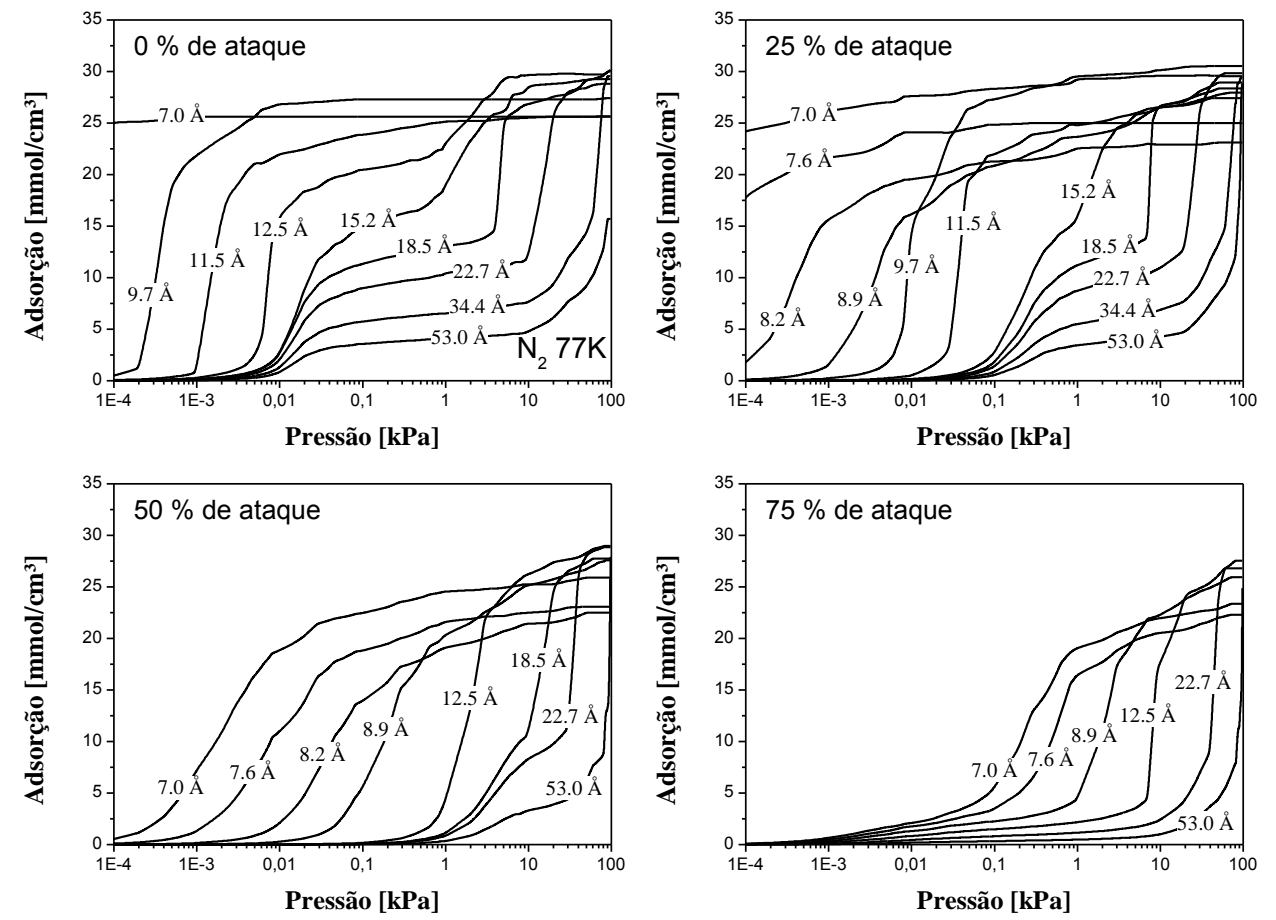

Figura 2 - Exemplos de isotermas locais de adsorção simuladas de $\mathrm{N}_{2}$ a $77 \mathrm{~K}$.

\subsection{Caracterização Textural com o Modelo Clássico}

Amostras de CA: seis amostras de CA foram caracterizadas: Maxsorb, WV-1050, Norit-R1, BPL e dois carbonos sintetizados a partir de casca de coco (W1 e W2). Inicialmente utilizou-se apenas o kernel-A, e fator de regularização calculado de 0,02 para todas as amostras. As PSDs obtidas encontram-se na Figura 3.

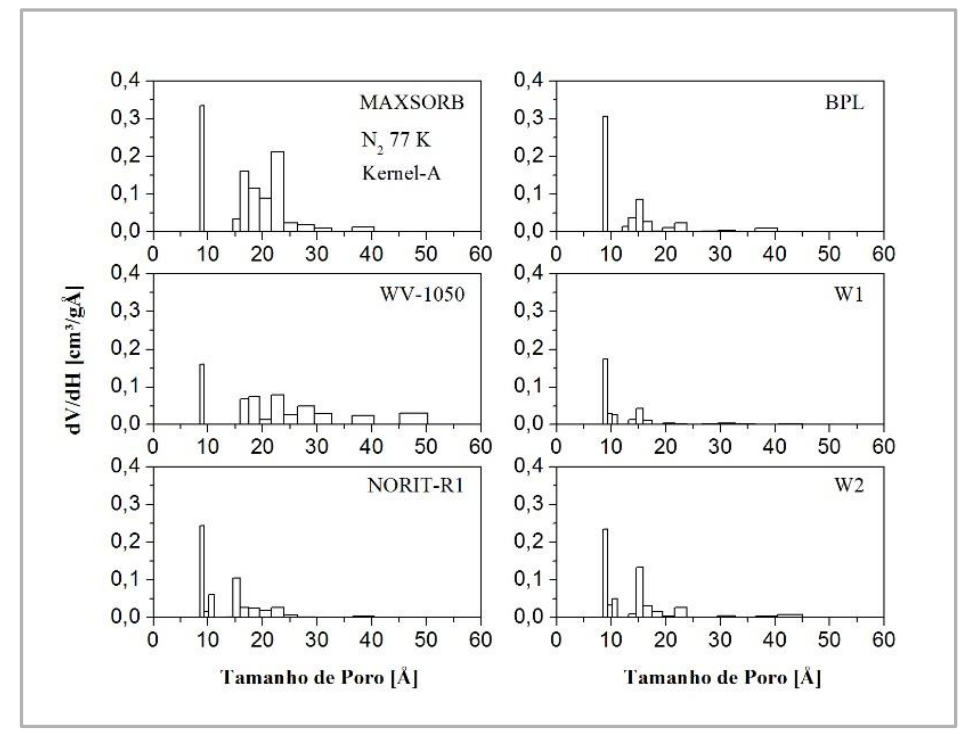

Figura 3 - PSDs obtidas com a deconvolução do kernel-A. 
De maneira geral as PSDs obtidas com nitrogênio a $77 \mathrm{~K}$ foram condizentes com as características texturais conhecidas das amostras: Maxsorb e WV-1050 - de micro a mesoporosos; Norit-R1, BPL, W1 e W2 - predominantemente microporoso. Nota-se uma semelhança entre as PSDs das amostras W1 e W2, com maior volume para a amostra mais ativada (W2). Isto ocorreu devido ao fato de serem amostras de mesmos material precursor.

Os ajustes às isotermas experimentais, Figura 4, apresentaram-se inconsistentes, sobretudo para as amostras com maior capacidade adsortiva (Maxsorb, WV-1050). É possível observar um perfil sinuoso em pressões inferiores a $10 \mathrm{kPa}$. Tal deficiência pode ser explicada pela heterogeneidade superficial presente nestas amostras e não avaliadas com o kernel-A.

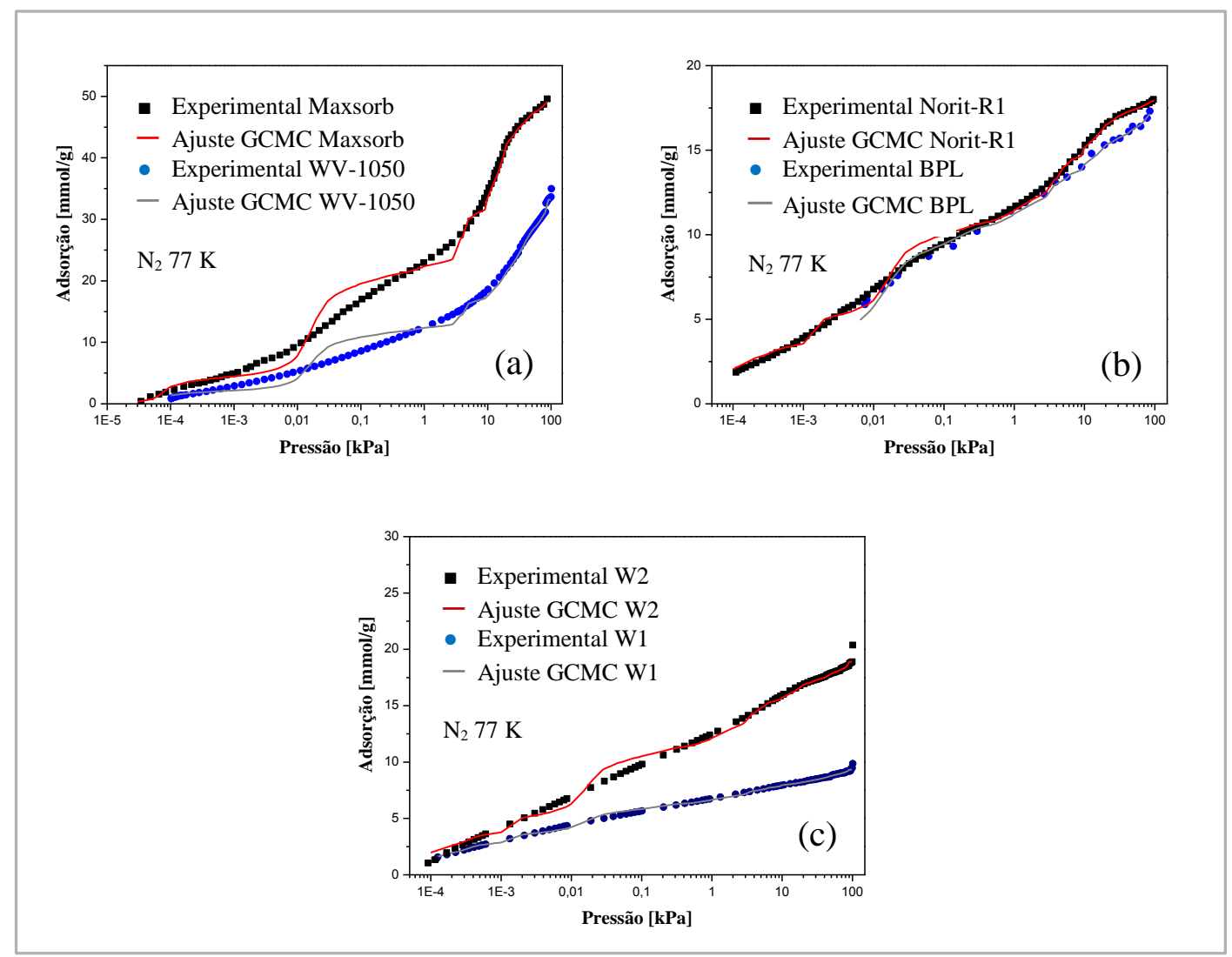

Figura 4 - Ajustes às isotermas experimentais com o kernel A: Maxsorb e WV 1050 (a), Norit-R1 e BPL (b) e W1 e W2 (c).

\subsection{Caracterização Textural com o Modelo Heterogêneo}

Maxsorb e WV-1050: A PSD obtida com o kernel-B para as amostras Maxsorb e WV-1050, Figura 5 (a) e (b), apresentaram grandes volumes de poros atacados, indicando que são amostras altamente heterogêneas. Observe que as PSDs obtidas com modelos heterogêneos são representadas por um gráfico composto por quatro PSDs, estas fazem parte de uma PSD única que foi separada desta forma para melhor visualização.

Os ajustes obtidos, Figura 5 (c) e (d), apresentaram grande proximidade com as isotermas experimentais em toda a faixa de pressão. Se comparado ao fraco ajuste obtido com o kernelA, evidencia-se a importância de modelos heterogêneos para modelar esta amostra. 

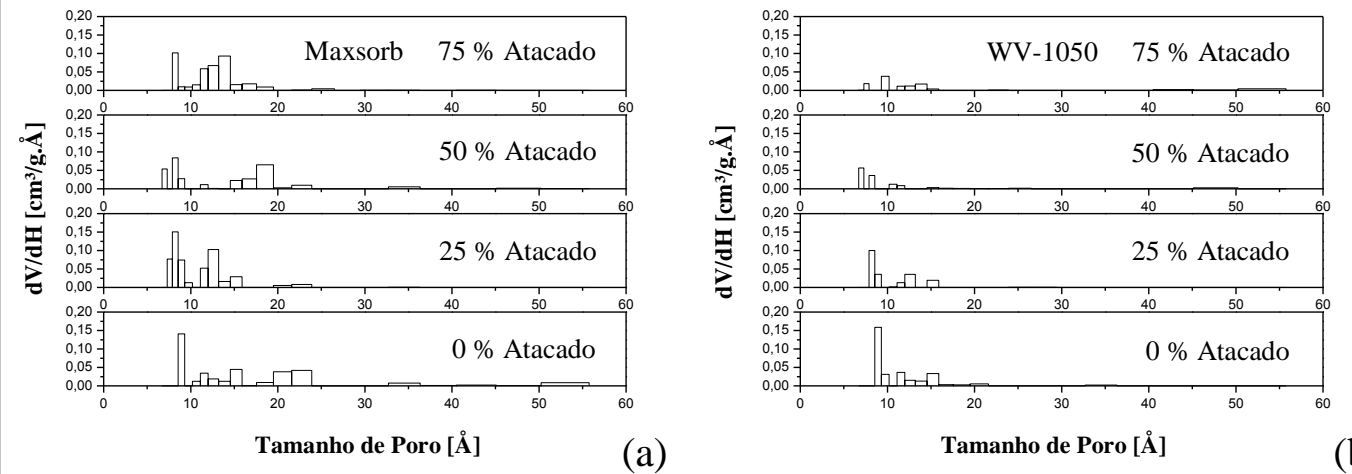

(a)

(b)
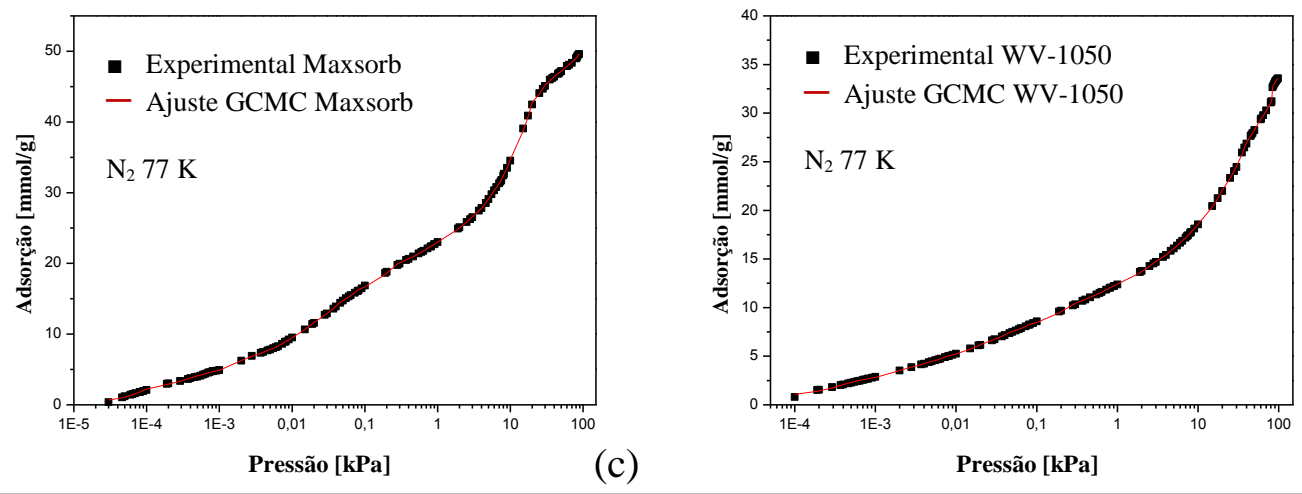

(d)

Figura 5 - PSDs das amostras Maxsorb (a) e WV-1050 (b) e ajuste às isotermas experimentais do Maxsorb (c) e WV-1050 (d), obtida com a deconvolução do kernel-B.

Norit-R1 e BPL: A PSD obtida para o Norit-R1, Figura 6 (a), e para o BPL, Figura 6 (b), apresentaram considerável volume de poros atacados, apresentando reduzido volume de poros heterogêneos na região de $75 \%$ de ataque. Este resultado condiz com o fato de serem amostras heterogêneas, porém menos heterogêneas que o Maxsorb e o WV-1050. Os ajustes às isotermas experimentais, Figura 6 (c) e (d), apresentaram-se satisfatórios.

W1 e W2: As PSDs obtidas para estas duas amostras, Figura 7 (a), mostraram-se condizentes com o grau de ativação das amostras, menos ativadas que as amostras Norit-R1 e BPL, e com isso menos heterogêneas. Novamente percebe-se uma semelhança entre as PSDs destes dois carbonos, com maior volume de poros heterogêneos para a amostra W2. Os ajustes obtidos para as duas amostras, Figura 7 (b), apresentaram boa qualidade, com destaque para a evolução do ajuste à isoterma do W2. Este resultado indica maior dependência da amostra W2 com o modelo heterogêneo, uma vez que esta amostra é mais ativada.

Erros Residuais: Os erros residuais dos ajustes, veja a Equação 2, foram avaliados e organizados na Tabela 3. Observa que com o kernel-A o valor do erro é proporcional à capacidade adsortiva, enquanto que com o kernel-B todos os erros apresentaram a mesma ordem de grandeza. Tal resultado reforça a necessidade de considerar fatores de heterogeneidade no modelo de CA, principalmente para amostras com maior capacidade adsortiva. 

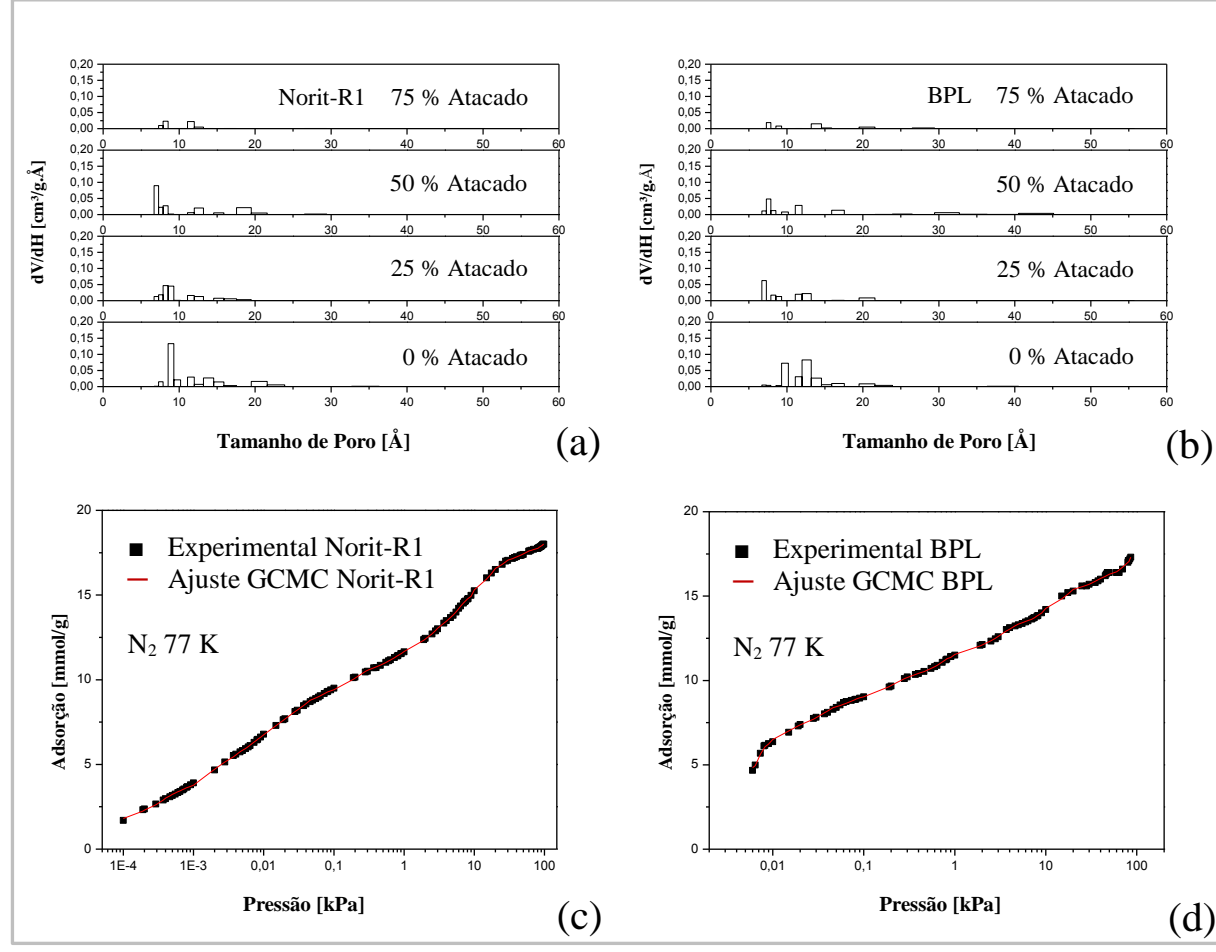

Figura 6 - PSDs das amostras Norit-R1 (a) e BPL (b) e ajuste às isotermas experimentais do Norit-R1 (c) e BPL (d), obtidos com a deconvolução do kernel-B.
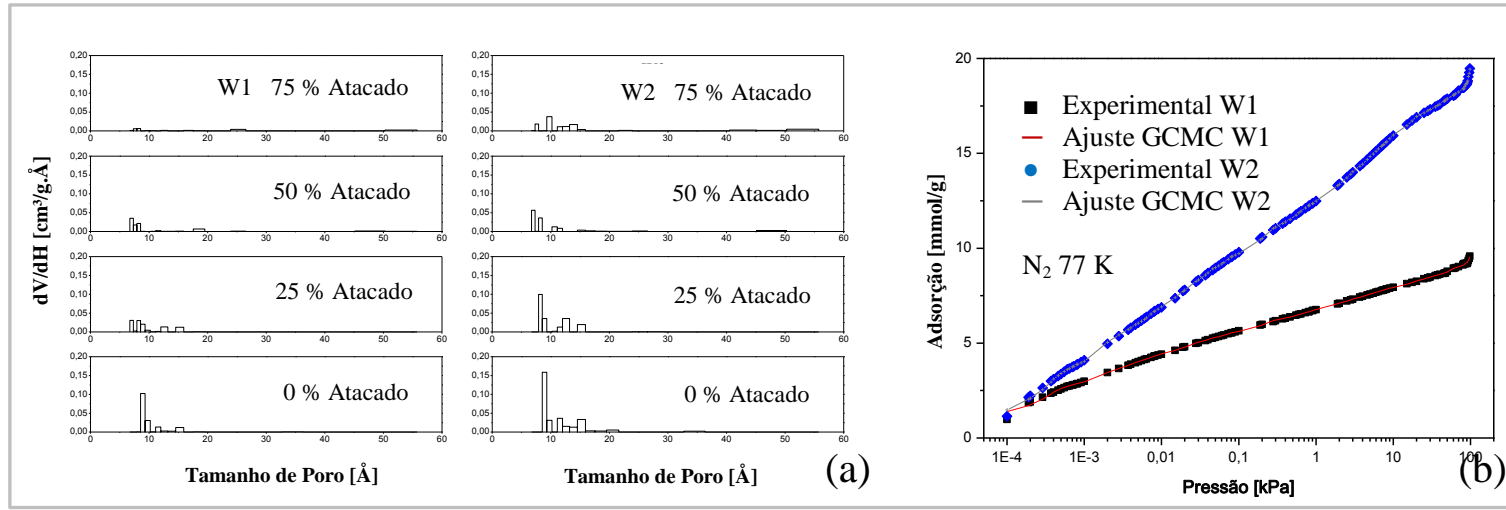

Figura 7 - PSDs (a) e ajustes às isotermas experimentais (b) para amostras W1 e W2.

Tabela 3 - Erros residuais dos ajustes obtidos com os kernels A e B

\begin{tabular}{ccc|ccc}
\hline Amostra & kernel-A & kernel-B & Amostra & kernel-A & kernel-B \\
\hline Maxsorb & 16,62 & 0,97 & BPL & 2,79 & 0,55 \\
WV-1050 & 12,45 & 0,55 & W1 & 1,54 & 0,38 \\
Norit-R1 & 3,49 & 0,38 & W2 & 4,74 & 0,38 \\
\hline
\end{tabular}

\section{CONSIDERAÇÕES FINAIS}

Foram realizadas caracterizações texturais de seis amostras de carbono ativado, com diferentes graus de ativação, e em todos os casos o ajuste obtido pela deconvolução da coleção 
de isotermas de poro perfeitos, kernel-A, mostrou-se insatisfatório. O kernel-B, contendo isotermas de poros imperfeitos, mostrou-se mais ajustou as isotermas experimentais das seis amostras com grande precisão. Os resultados obtidos com o modelo atacado demonstram a necessidade de modelos mais realistas para simular amostras de carbono ativado, sobretudo as mais heterogênea.

Ao analisar a variação entre os ajustes com e sem poros imperfeitos, pode-se observar que quanto maior a capacidade adsortiva da amostra, maior a sensibilidade ao modelo de poros imperfeitos. Tal fato reforça a hipótese de uma relação direta entre heterogeneidade e capacidade adsortiva. Por fim os erros residuais dos ajustes foram quantificados, revelando uma grande discrepância entre os erros gerados com o kernel-A e com o kernel-B.

\section{REFERÊNCIAS BIBLIOGRÁFICAS}

ALLEN, M. P.; TILDESLEY, D. J. Computer simulation of liquids. New York: Clarendon Press Oxford, 1991.

BIGGS, M.J.; BUTS, A. Virtual porous carbons: what they are and what they can be used for. Mol. Sim., v. 32, p. 579-593, 2006.

DAVIES, G.M.; SEATON, N.A.; VASSILIADIS, V.S. Calculation of Pore Size Distributions of Activated Carbon from Adsorption Isotherms. Langmuir, v. 15, p. 8235-8245, 1999.

LUCENA, S.M.P.; PAIVA, C.A.S.; SILVINO, P.F.G.; AZEVEDO, D.C.S.; CAVALCANTE JR., C.L. The effect of heterogeneity in the randomly etched graphite model for carbon pore size characterization. Carbon, v. 48, p. 2554-2565, 2010.

MARSH, H.; RODRÍGUEZ-REINOSO, F.. Activated Carbon. Amsterdam: Elsevier Science \& Technology Books, 2006.

SEATON, N.A.; FRIEDMAN, S.P.; MACELROY, J.M.D.; MURPHY, B.J. The Molecular Sieving Mechanism in Carbon Molecular Sieves: A Molecular Dynamics and Critical Path Analysis. Langmuir, v. 13, p. 1199-1204, 1997.

TAN, Z.; GUBBINS, K.E.J. Selective Adsorption of Simple Mixtures in Slit Pores: A Model of Methane-Ethane Mixtures in Carbon. Phys. Chem., v. 96, p. 845-854, 1992.

WILSON, J.D. Statistical approach to the solution of first-kind integral equations arising in the study of materials and their properties. Journal of Materials Science, v. 27, p. 3911-3924, 1992. 